Full Proceeding Paper

\title{
MODELING OF LIGHT ILLUMINATION FIELD ON MICROEXPRESSION FOR FACE RECOGNITION APPLICATION
}

\author{
ANKUSH RAI, JAGADEESH KANNAN R \\ School of Computing Science and Engineering, VIT University, Chennai, Tamil Nadu, India. Email: ankushressci@gmail.com
}

Received: 28 December 2016, Revised and Accepted: 10 May 2017

\begin{abstract}
Objective: Face recognition algorithms often suffer the problem of varying light illumination while extracting the feature points and thereby degrades the recognition rate. Although past techniques in the same area often require manual setting of thresholding parameters.

Methods: In this study, the presented technique solves the problem of variation in light illumination by modeling the light field on facial microexpression for improving the performance of the face recognition. The performance of the method is compared with the other techniques based
\end{abstract} on two benchmark datasets, namely, CMU pose, illumination, and expression (PIE), and multi-PIE.

Results and Conclusion: Upon compraission the error rates has been minimal from rest of the methods as only unique match points is selected by the algorithm and latter used it to render over the other similar images which give the higher correct match acceptance rate in nominal rate.

Keywords: Facial recognition, Face detection, Algorithm design and analysis.

(C) 2017 The Authors. Published by Innovare Academic Sciences Pvt Ltd. This is an open access article under the CC BY license (http://creativecommons. org/licenses/by/4. 0/) DOI: http://dx.doi.org/10.22159/ajpcr.2017.v10s1.19653

\section{INTRODUCTION}

In the past, several studies had been conducted to address the problem of face recognition. Such methods enable fast classification, high accuracy, and lower false recognition rate with several other advantages of ranging from high performance to automating the processing operations; which in turn has increased its applicability in several of the practical domains.

In face matching scenario, the recognition of the two images for the same person is specifically a daunting task when the image is taken under different light illumination field. Primarily, there are three sets of classes to approach this problem:

1. The first relies on building a classifier to operate upon image features with variant illumination. Principal methods such as local binary patterns, local gabor binary patterns, and local ternary patterns [1-3]

2. The second revolves around the idea of eliminating the effects of illumination from the images by normalizing out the reflectance field (example: Lambertian). In this approach, the specific image processing method is adopted to stepwise empirically carve the desired output. Among those popular are categorized as quotient image, generalized quotient image, total variation model, and tan and triggs lighting normalization [2-6].

3. Lastly, the last category in this approach involves the generation of synthetically relighted images to generalize the light illumination from the given image, so as to match all the illumination variants within the probe set. This enables easy matching of the enrolled images with that of the given image in the simulated lighting condition (using Phong model) to find the close match among the test gallery. Such method increases the probability of matching the facial image with at least one of the several images from the augmented illuminated image. The deviants three-dimensional (3D) morphable models method is the popular one includes the cited [7-9]. It involves fitting the model of 3D shape over the model of texture to give an optimized matching output of gallery augmentation. Although its effectiveness it suffers major drawback which includes a cumbersome process of manual quality relighting.
As a resultant of it methods such as Sim and Kanade [10] and Lee and Moghaddam [11] does not even require explicit 3D shape fitting models and hence in practice are claimed to be computationally robust in practice. The second and the third method is the inverse of each other as in one attempt is made to remove illuminating effects whereas in other efforts has been made to synthesize the reflectance condition, though none can be previously controlled.

Given that this studies has already accomplished a majority of work in detection and recognition of faces but are often prone to several of the real-time variations such as continuously varying lighting scheme, chromatic aberration, and low lighting environments. Therefore, rather than adding a yet another patching techniques based on the current literature of classifiers for face recognition, we have presented a novel and simplistic algorithm for easy face detection using multiwavelet scaling transformation which shall have the characteristic of adaptive learning scheme. This enables it to model the problem of varying light illumination field based upon that it will simulate the lighting to adjust and continues to give a constant high performance in a challenging scenario. Latter in the study, we will demonstrate the superiority of the proposed method over the wide variety of existing classifier methods for face detection; while using the two datasets, that is, mainly multipose, illumination, and expression (PIE) and CMU PIE $[12,13]$.

\section{Experimental setup}

The proposed model is implemented using MATLAB R2012a under Windows platform. The experiments are conducted on the machine with hardware configurations of Intel's third generation 8-core microprocessor with Nvidia 630 graphic card, 2 GB RAM giving a fine clocking speed of $2.7 \mathrm{GHz}$. The consolidated database available online at Yale University with the name of extended Yale B dataset is used in the study to read the performance of the proposed algorithm. Since it contains a large variety of point-source lit images [14]. Furthermore, additionally, we used the images from CMU PIE and Multi-PIE face database as represented in Table 1 as test sets $[12,13]$. The images used in the study consist of frontal and side pose while accompanying neutral to sad, happy, and awkward microexpressions. The measures were taken to limit the number of false acceptance as the training 
gallery is formed only with the relative images. Finally, the algorithm is also tested on a different range of lighting scheme from low to high, and the results are shown in latter sections.

\section{METHODOLOGY: PYRAMIDAL SCALAR ASSOCIATIVE ALGORITHM (PSA)}

Our work is based on the idea of the finding the topology of the features of a facial image and then coding it in sequence with the cascaded neural network; since in the previous study such attempt has already been quite successful in decoding the face recognition patterns involved with human brain and then training the neural nets to mimic such process [16-18]. Before we begin with the transformation of wavelets to break down the features of the facial image in logical association with each other, it is required that the given image is to be pre-processed such that the wavelets of the broken image shall be easy to perform computational operations with optimized values. Such that the given RGB standard image is to be normalized in rg scheme (Fig. 1). Thus, using the following algorithm for pixel normalization is:

Algorithm: RGB normalization to rg scheme

Input: $\mathrm{m} \times \mathrm{n} \mathrm{RGB}$ standard Image

Where, $m$ and $n$ are the row and column of the given image.

Output: rg normalized color scheme

Loop: for i to $\mathrm{m}$

Loop: for $\mathrm{j}: \mathrm{n}$

$$
\begin{aligned}
& r_{i, j}=\frac{R}{(R+G+B)} \\
& g_{i, j}=\frac{G}{(R+G+B)}
\end{aligned}
$$

end

end

Table 1: Enlisted database used in the experiment

\begin{tabular}{lllll}
\hline Database & Subjects & $\begin{array}{l}\text { Images per } \\
\text { person }\end{array}$ & $\begin{array}{l}\text { Repeated } \\
\text { micro-expression } \\
\text { per person }\end{array}$ & Total \\
& & & 2 & 160 \\
\hline Extended Yale B & 40 & 4 & 3 & 490 \\
CMU PIE & 70 & 7 & 6 & 4788 \\
Multi-PIE & 342 & 14 & &
\end{tabular}

PIE: Pose, illumination, and expression
As due to the compactness of the given facial image, the distribution of the skin color can be approximated to $\mathrm{M} \sim \mathrm{N}$ using Gaussian distribution. Thus, the segmented pixels representing skin color can be segmented in the form of a vector as:

$$
\text { If } \begin{aligned}
\left(\gamma_{M} \leq \sum_{M_{1}}\right) & \\
& P_{\text {skin }}=P(\operatorname{skin} \mid r g, N)
\end{aligned}
$$

else

$$
\mathrm{P}_{\text {background }}=\left(\text { background } \mid \mathrm{rg}, \gamma_{\mathrm{M}}\right)
$$

Where, the value $\mathrm{M}$ represents the model of skin color, which is embarked as low-intensity pixels after the pre-processing. $\gamma_{\mathrm{M}}$ and $\Sigma_{\mathrm{M}}$ are the mean and covariance of the pixel distribution based on intensities in rg color scheme after pre-processing.

Now, that we have the segmented pixels sets; it is required to frame up an adaptive algorithm to code facial features and its orientation for both high- and low-resolution images. Therefore, we use the cascaded neural networks for the same. To form an associative pattern between the neighboring sets of pixel blocks the cascaded coding of training sets requires three vectors:

a. Bit rate of transmitting the displacement vector field

b. Bit rate for sequencing connectomes of information tree

c. The error rate in learning.

Given the segmented datasets derivable from the above algorithm $P_{\text {skin }}$ in the form of each rg-color scheme is given as: $\left(\mathrm{x}_{1}, \mathrm{y}_{1}\right),\left(\mathrm{x}_{2}, \mathrm{y}_{2}\right), \ldots$, $\left(\mathrm{x}_{1}, \mathrm{y}_{\mathrm{l}}\right) \in \mathrm{P}_{\text {skin }} \times[1,0]$. Let $\mathrm{v}_{\mathrm{i}} \in \mathrm{V}$ be the set of displacement vectors for each of the neighboring nodes $n_{i \cdot}$ Such nodes represent the positioning of the feature sets. Therefore, to sequence the features of the facial image from the reconstructed rg-color scheme we use the following PSA algorithm; for which its face recognition process is depicted in Fig. 2.

Algorithm: PSA algorithm

Input: $\left(\mathrm{x}_{1}, \mathrm{y}_{1}\right),\left(\mathrm{x}_{2}, \mathrm{y}_{2}\right), \ldots,\left(\mathrm{x}_{\mathrm{j}}, \mathrm{yj}\right) \in \mathrm{P}_{\text {skin }}$

Output: $\mathrm{P}_{\text {face }}(\mathrm{i}, \mathrm{j})$

Step 1: While $1 \leq \min \left(\sum_{\mathrm{i}=1}^{\mathrm{N}} \mathrm{D}\left(\mathrm{v}_{\mathrm{i}}, \mathrm{n}_{\mathrm{i}}\right)\right) / \mathrm{D}$ is the displacement vector.

Step 2: Evaluate the feasible value of target nodes $R_{\text {target }}$ :

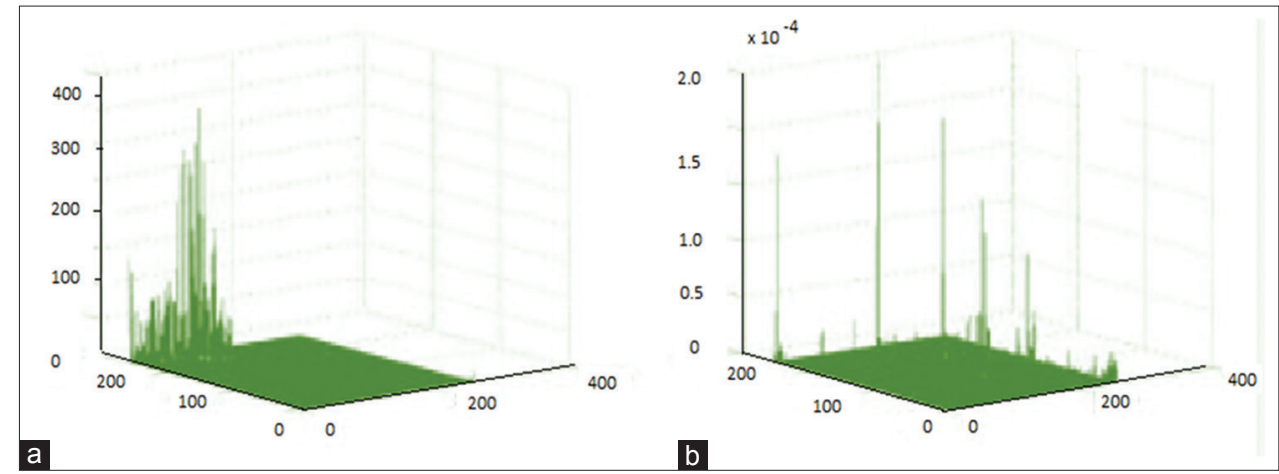

Fig. 1: The histogram plot of various sections of the image after pre-processing (a) shows that of the distribution of skin pixels $P_{\text {skin }}$ for the normalized rg-color scheme (b) shows that of the distribution of non-skin pixels or other background extracted pixels in pixel set $P$ 


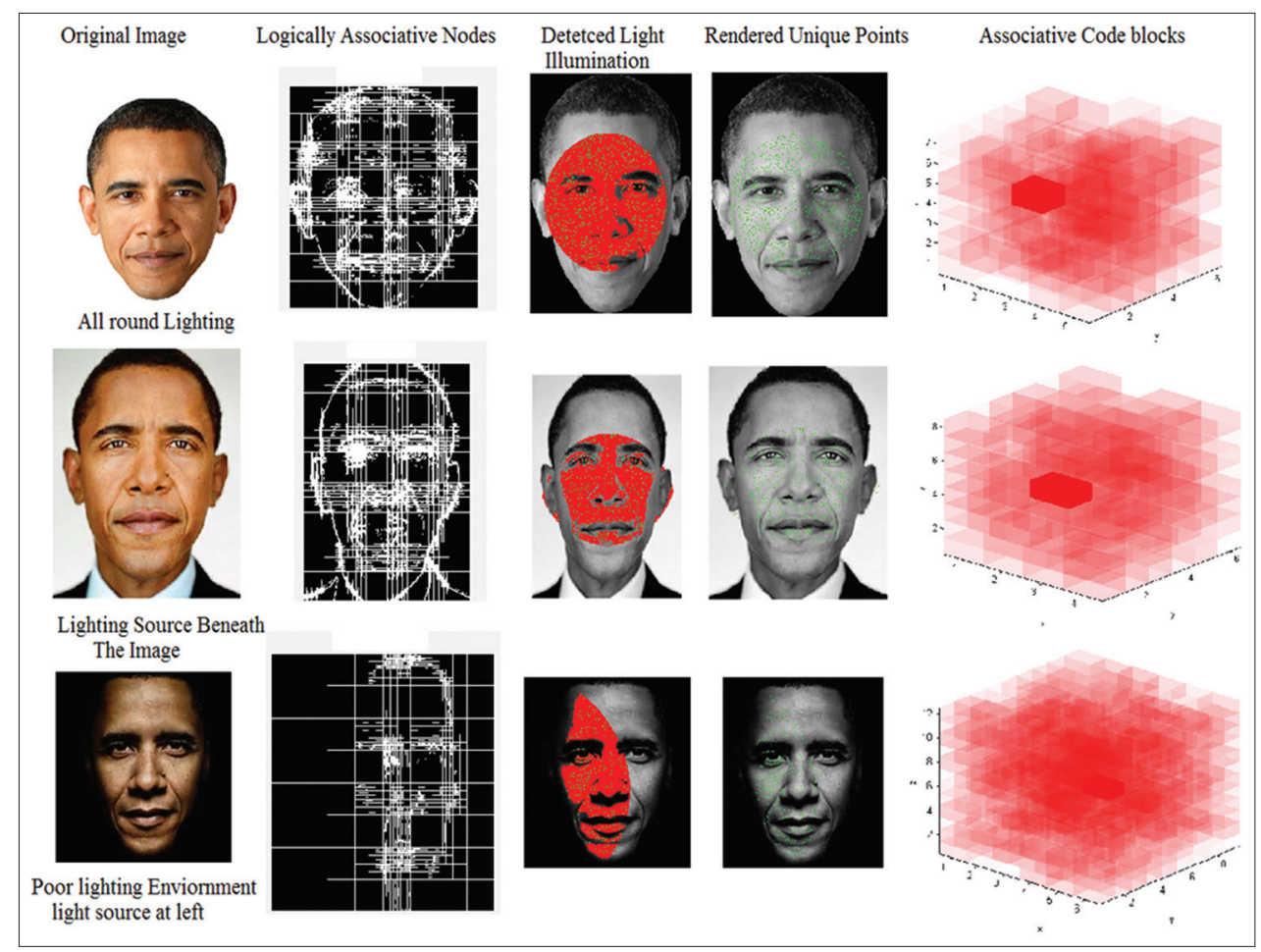

Fig. 2: The face recognition process using pyramidal scalar associative algorithm. In the left column, the original image is presented with different positioning of the light source. The second column represents the logically associated nodes formed in the due process. The third column represents the detected regions of light heavy illumination. The fourth column represents the rendered unique nodes extracted based on the logical association and the last column represents the three dimensional consolidated code blocks after the activation of cascaded neural network. As shown in the figure, the code block becomes clouded when there is poor lighting and though the face recognition is subjected to matching only the unique points discovered in the fourth column. Hence, reducing the chances of unnecessary matching points which has lower or none probability of finding it in similar facial images

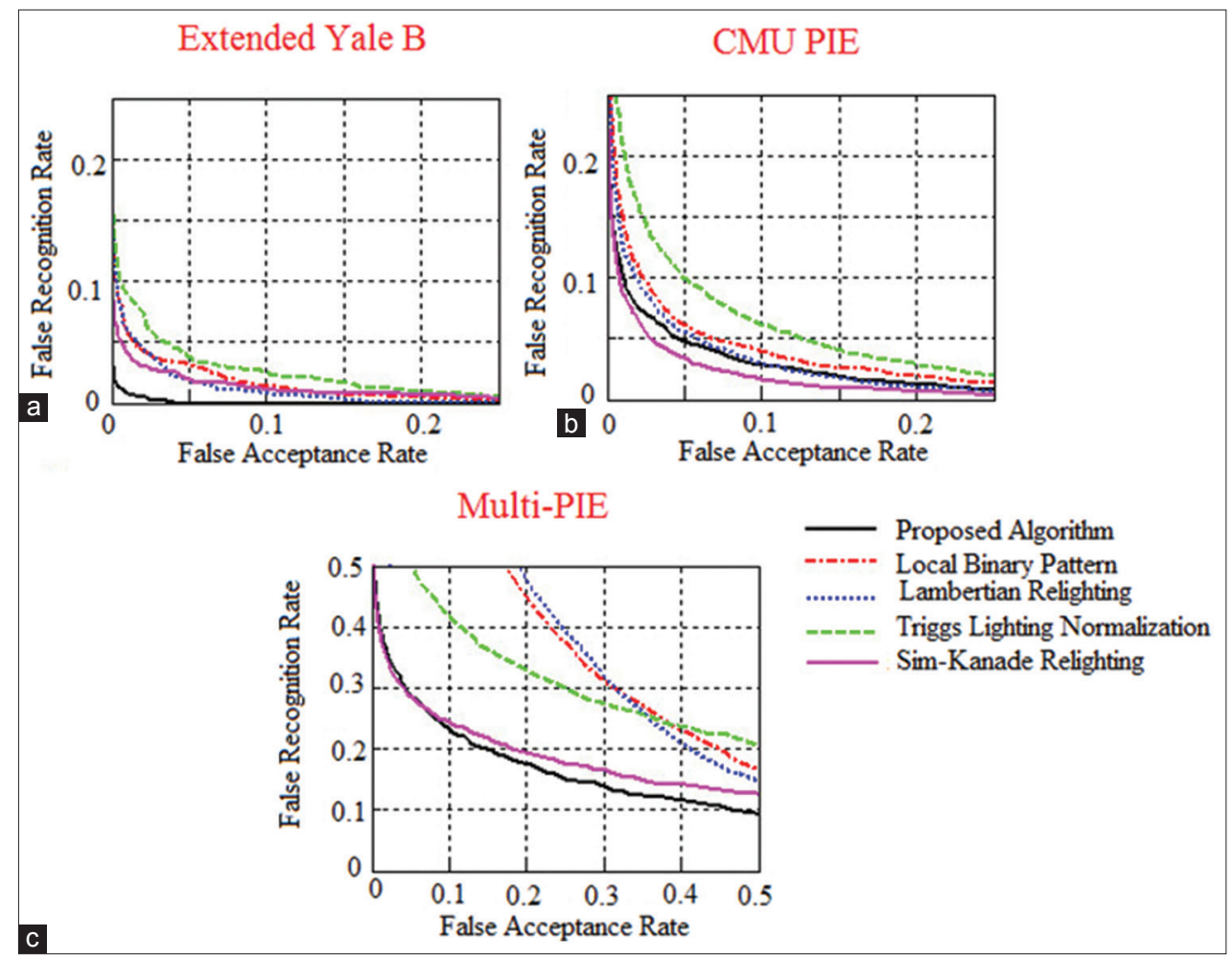

Fig. 3: (a-c) The comparison plot of the proposed pyramidal scalar associative algorithm with that of the other cited methods in the literature 


$$
\mathrm{R}_{\text {target }}=\sum_{\mathrm{i}=1}^{\mathrm{N}} \mathrm{D}\left(\mathrm{v}_{\mathrm{i}}, \mathrm{n}_{\mathrm{i}}\right)
$$

Step 3: Create target vectors for feasible neighboring nodes:

Loop: for 1 to $n$

$$
\sum_{i=1}^{N} R\left(v_{i}, n_{i}\right) \leq R_{\text {target }}
$$

Step 4: Using Lagrange multiplier $\lambda$ for the activation function of the cascaded neural network [15]:

$$
\operatorname{CC}(\mathrm{i}, \mathrm{j})=\sum_{\mathrm{i}=1}^{\mathrm{m}} \sum_{\mathrm{j}=1}^{\mathrm{n}} \operatorname{sgn}\left(\mathrm{D}\left(\mathrm{v}_{\mathrm{i}}, \mathrm{n}_{\mathrm{i}}\right)+\lambda \mathrm{R}\left(\mathrm{v}_{\mathrm{i}}, \mathrm{n}_{\mathrm{i}}\right)\right)
$$

end for loop

end while loop

Step 5: End process.

\section{CONCLUSION}

As shown in Fig. 3, the plot depicts the comparison of the several methods of face recognition relatively with the proposed PSA algorithm. The result is plotted between the two results, that is, false recognition rate and false acceptance rate. Here, the false acceptance rate represents the matching points chosen by the algorithm to be used for matching whereas false recognition rate is the matching points which are matched with the criteria chosen with the designated algorithms. As can be concurred from the above plot the error rates has been minimal from rest of the methods as only unique match points is selected by the algorithm and latter used it to render over the other similar images which give the higher correct match acceptance rate in nominal rate. This detection method gives us an advantage over the present existing methods which indulges in pointless computation and matching of the pixel sets with numerous data entries. Our method has reduced this redundancy to a higher limit and is promises to be useful in industrial applications. The issues with the other methods are that they heavily rely on the visual cues for face detection and thus are time-consuming. This method proposes a scalable algorithm with the cascaded neural network for logically associative defining nodes of the facial feature sets and thus the searching of match properties is reduced to a very fine scale. Furthermore, for lower resolution images PRA works fine from coarse to fine scales of visual cues in the context of skin color and textures. Recognition of facial images from different plane shall be pursued in the near future that will add depth to the current PSA algorithm.

\section{REFERENCES}

1. Ahonen T, Hadid A, Pietikainen M. Face description with local binary patterns: Application to face recognition. IEEE Pattern Anal Mach Intell 2006;28(12):2037-41.

2. Zhang W, Shan S, Gao W, Chen X, Zhang H. Local Gabor binary pattern histogram sequence (LGBPHS): A novel non-statistical model for face representation and recognition. International Conference on Computer Vision; 2005. p. 786-791.

3. Tan X, Triggs B. Enhanced local texture feature sets for face recognition under difficult lighting conditions. Analysis and Modeling of Faces and Gestures; 2007. p. 168-182.

4. Shashua A, Raviv TR. The quotient image: Class based re-rendering and recognition with varying illuminations. IEEE Pattern Anal Mach Intell 2001;23(2):129-139.

5. Wang H, Li S, Wang Y. Generalized quotient image. In: Computer Vision and Pattern Recognition; 2004.

6. Chen T, Yin W, Zhou XS, Comaniciu D, Huang T. Total variation models for variable lighting face recognition. IEEE Pattern Anal Mach Intell 2006:28:1519-24.

7. Blanz V, Vetter T. Face recognition based on fitting a 3D morphable model. IEEE Pattern Anal Mach Intell 2003;25(9):1063-74.

8. Wang Y, Zhang L, Liu Z, Hua G, Wen Z, Zhang Z, et al. Face relighting from a single image under arbitrary unknown lighting conditions. IEEE Pattern Anal Mach Intell 2009;31(11):1968-84

9. Zhang L, Samaras D. Face recognition from a single training image under arbitrary unknown lighting using spherical harmonics. IEEE Pattern Anal Mach Intell 2006;28(3):351-63.

10. Sim T, Kanade T. Combining models and exemplars for face recognition: An illuminating example. CVPR Workshop on Models versus Exemplars in Computer Vision; 2001.

11. Lee KC, Moghaddam B. A practical face relighting method for directional lighting normalization. Analysis and Modelling of Faces and Gestures; 2005.

12. Sim T, Baker S, Bsat M. The CMU pose, illumination, and expression database. IEEE Pattern Anal Mach Intell 2003;25(12):1615-8

13. Gross R, Matthews I, Cohn J, Baker S, Kanade T. The CMU MultiPose, Illumination, and Expression (Multi-PIE) Face Database. Tech Rep TR-07-08, CMU; 2007.

14. Lee KC, Ho J, Kriegman DJ. Acquiring linear subspaces for face recognition under variable lighting. IEEE Pattern Anal Mach Intell 2005;27(5):684-98.

15. Barmpoutis A, Kumar R, Vemuri BC, Banerjee A. Beyond the lambertian assumption: A generative model for apparent BRDF fields of faces using anti-symmetric tensor splines. Computer Vision and Pattern Recognition; 2008.

16. Rai A. Attribute based level adaptive thresholding algorithm (ABLATA) for image compression and transmission. J Math Comput Sci 2014;12:211-8.

17. Rai A. An introduction of smart self-learning shell programming interface. J Adv Shell Program 2015;12:3-6.

18. Rai A. Dynamic data flow based spatial sorting method for GPUs: Software based autonomous parallelization. Recent Trends Parallel Comput 2014;11:15-8 